\title{
The prognostic value of preoperative FDG-PET/CT metabolic parameters in cervical cancer patients
}

Vikram Rao Bollineni ${ }^{1,2,3^{*}}$ (D), Sigmund Ytre-Hauge ${ }^{4,5}$, Ankush Gulati ${ }^{4,5}$, Mari K. Halle ${ }^{1,2}$, Kathrine Woie', Øyvind Salvesen ${ }^{6}$, Jone Trovik ${ }^{1,2}$, Camilla Krakstad ${ }^{1,2}$ and Ingfrid S. Haldorsen ${ }^{4,5^{*}}$

\section{* Correspondence:}

bollinenivr@yahoo.com; vikram.

bollineni@uib.no; ingfrid.haldorsen@ uib.no

${ }^{1}$ Department of Obstetrics and Gynecology, Haukeland University

Hospital, Bergen, Norway

${ }^{4}$ Mohn Medical Imaging and

Visualization Centre (MMIV),

Department of Radiology,

Haukeland University Hospital,

Bergen, Norway

Full list of author information is

available at the end of the article

\begin{abstract}
Background: To explore quantitative metabolic and microstructural primary tumour parameters at pretreatment FDG-PET/CT and diffusion-weighted-magnetic resonance imaging (DW-MRI) in relation to clinical FIGO stage and outcome in uterine cervical cancer patients.

Methods: Fifty three patients with histopathologically verified cervical carcinoma with clinical FIGO stage IB1-IVA were subjected to FDG-PET/CT and subgroup also DW-MRI $(n=30)$ prior to treatment. Measurements from the FDG-PET/CT comprised lesion maximum-standardised uptake value (SUV $\left.V_{\text {max }}\right)$, total lesion glycolysis (TLG) and metabolic tumour volume (MTV). In MR images longest-tumour-diameter (MRI-LD), tumour volume (MRI-TV) and mean tumour apparent-diffusion-coefficient ( $A D C_{\text {mean }}$ ) value were measured. FDG-PET/CT parameters were explored in relation to clinical prognostic factors at diagnosis and progression/recurrence free survival, and compared with the MRI parameters.
\end{abstract}

Results: The metabolic tumour parameters TLG and MTV were highly positively correlated to MRI-LD and MRI-TV ( $r_{s}=072-0.82 ; p<0.001$ for all), whereas tumour SUV $\max$ was only moderately correlated to MRI-LD $\left(r_{s}=0.29 ; p \leq 0.04\right)$ and MRI-TV $\left(r_{s}=0.36 ; p \leq 0.01\right)$. High tumour TLG, MTV, MRI-LD and MRI-TV predicted advanced FIGO stage, whereas high tumour SUV max $_{\text {ax }}$ did not. No significant correlations were observed between tumour $\mathrm{ADC}$ mean and the other imaging parameters or FIGO stage. High primary tumour MTV ( $\geq 56.7 \mathrm{~mL})$, high TLG $(\geq 412 \mathrm{~g})$ and large MRI-TV $(\geq 36 \mathrm{~mL})$ predicted reduced progression/recurrence free survival yielding corresponding hazard ratios $[\mathrm{HR}]$ of $7.8(P=0.002), 6.9(P=0.004)$ and $4.6(P=0.022)$, respectively. Also advanced FIGO stage $(\geq I I A)$ was associated with reduced progression/recurrence free survival with HR of $6.9(P=0.004)$. In multivariable analysis, advanced FIGO stage $(\geq I I A)$ and high MTV $(\geq 56.7 \mathrm{~mL})$ were independent prognostic factors with adjusted HRs of $5.5(P=0.020)$ and $7.8(P=0.025)$, respectively.

Conclusion: High MTV at pre-treatment FDG-PET/CT and high clinical FIGO stage independently predict reduced progression/recurrence free survival in cervical cancer patients.

Keywords: Cervical cancer, FDG-PET/CT, DW-MRI, MTV, TLG 


\section{Background}

Cervical cancer is one of the most frequently occurring gynaecologic malignancies and the third most common cause of death among patients with gynaecologic cancers in the United States (Howlader et al., 2011). Cervical cancer has until recently been clinically staged according to the International Federation of Gynaecology and Obstetrics (FIGO) classification system (Benedet et al., 2000). Very recently (2017), the European Society of Gynaecological Oncology (ESGO) in collaboration with the European Society for Radiotherapy and Oncology (ESTRO) and the European Society of Pathology (ESP) introduced new guidelines on the management of invasive cervical cancer according to TNM staging (Cibula et al., 2017). However, clinical FIGO stage should still be reported in addition to TNM staging. Diagnostic imaging with pelvic magnetic resonance imaging (MRI) and fluorodeoxyglucose (FDG)-positron emission tomography/computed tomography (PET/ CT) is increasingly performed pretherapeutically for assessment of local tumour extent, lymph node metastases, and distant spread. Furthermore, pretreatment FDG-PET/CT yields several metabolic tumour parameters, which may aid in the prediction of lymph node metastases, advanced stages, and poor outcome in cervical cancer (Mirpour et al,, 2013; RSM et al., 2016; Lv et al., 2014; Sironi et al., 2006; Choi et al., 2006). However, the added clinical value of these imaging biomarkers for cervical cancer and how these markers may be integrated into biomarker-guided treatment algorithms are largely unknown.

Diffusion-weighted MRI (DW-MRI) depicts microstructural properties of the tissue, and low apparent diffusion coefficient (ADC) values indicate the restriction of extracellular water mobility, which is typically observed in highly dense tissues (e.g. tumours and metastatic lymph nodes) and in highly fibrotic tissues (Le Bihan, 2013). Low tumour ADC value in cervical cancer is reportedly associated with high FIGO stage, large tumour size, and lymph node metastasis and is a poor prognostic factor (McVeigh et al., 2008; Nakamura et al., 2012; Miccò et al., 2014; Bollineni et al., 2015). The association between microstructural alterations depicted by DW-MRI and metabolic tumour changes observed on PET/CT is largely unknown. Furthermore, the most robust imaging biomarker with which to accurately define pretreatment tumour extent and for prognostication in cervical cancer patients is yet to be identified.

This study explored the relationship of quantitative metabolic and microstructural primary tumour parameters determined from pretreatment FDG-PET/CT and DW-MRI with clinical FIGO stages and outcomes in cervical cancer patients. Additionally, this study explored the correlations between primary tumour parameters derived from FDG-PET/CT and DW-MRI.

\section{Methods}

Patients

This study was conducted according to institutional review board-approved protocols, and informed consent was obtained from all patients. The recruited study patients diagnosed as having cervical cancer were all treated at the same university hospital, a European Society for Gynaecologic Oncology-accredited training centre for gynaecologic oncology that serves a population of approximately 1 million. The medical records of all patients diagnosed as having cervical cancer from October 2010 to December 2014 at the hospital were reviewed retrospectively to identify patients who had 
undergone pretreatment FDG-PET/CT $(n=53)$. Among them, a subgroup of 51 patients had also routinely undergone pelvic MRI, which included DW-MRI in 30/51 patients. The 53 patients who constituted the entire study sample had a median age of 50 years and were diagnosed as having clinical FIGO stage IB1-IVA cervical cancer (Table 1). Patient workup included the complete blood count, physical and gynaecological examinations, tests at baseline, and histological tumour biopsy confirming the diagnosis. Follow-up data on tumour progression/recurrence were collected from patient records. Progression-free survival was defined as the duration from the start of first-line treatment to progression of the disease (based on imaging findings), and recurrence-free survival was defined as the duration from the start of first-line treatment to the date of first detected recurrence (local or distant). The last date of follow-up was February 2016, and the mean (range) follow-up period for survivors was 25 months (range, 6-52 months).

\section{FDG-PET/CT}

FDG-PET/CT scans were obtained at the Department of Nuclear Medicine and Molecular imaging of the University Hospital Bergen on a Siemens mCT machine (Biograph 40 True Point scanner) according to the guidelines of the European

Table 1 Patient demographics and clinico-pathological characteristics

\begin{tabular}{ll}
\hline Patient demographics and histopathological findings & \\
\hline Variable & Number of patients (\%) \\
\hline Total patients & 53 \\
Median age (range) & $50(26-84)$ \\
FIGO stage & \\
IB1 & $7(13)$ \\
IB2 & $5(9)$ \\
IIA1 & $1(2)$ \\
IIA2 & $2(4)$ \\
IIB & $21(39)$ \\
IIIA & $1(2)$ \\
IIIB & $13(24)$ \\
IV & $1(2)$ \\
IVA & $1(2)$ \\
Histology & \\
SCC & \\
AC & $42(80)$ \\
Other & $7(13)$ \\
Progression/recurrence & $4(7)$ \\
Yes & \\
No & $10(19)$ \\
Alive & \\
Without evidence of disease & $13(24)$ \\
With evidence of disease & $40(76)$ \\
Death from disease & \\
\hline Abbrvations:FGO & \\
\hline
\end{tabular}

Abbreviations: FIGO International Federation of Gynaecology and obstetrics, SCC Squamous cell carcinoma, AC Adenocarcinoma 
Association of Nuclear Medicine (Boellaard et al., 2015).Measurement of blood glucose level with finger-stick before tracer injection to confirm an acceptable blood sugar level ( $<11 \mathrm{mmol} / \mathrm{l}$ ) after an overnight fast of minimum 6 h. (Lai et al., 2017)F-FDG (3 MBq/ kg bodyweight) was given intravenously 60-120 min (during which the patients rested in a semi dark, temperate room) before the PET/CT scan covering the skull to the proximal thigh. The PET data were reconstructed with high definition, ordered-subsets expectation maximization using 3 iterations, 21 subsets and 8-mm Gaussian post processing filter and had a spatial resolution of $2.04 \times 2.04 \times 2 \mathrm{~mm}^{3}$.

\section{MRI}

Pelvic MRI was performed at six different hospitals in Western Norway as part of routine diagnostic work-up. The MR scanners were from different manufacturers, but all on 1.5 Tesla scanners. The employed imaging protocols and the scanning parameters varied between centres and scanners, but all were dedicated pelvic protocols and were largely in accordance with European Society of Urogenital Radiology (ESUR) guidelines for staging of uterine cancer with MRI (Balleyguier et al., 2011). The DWI-MRI consisted of low b-value images (b-values in the range of 0 to $50 \mathrm{~s} / \mathrm{mm}^{2}$ ) and high b-value images (b-values in the range of 800 to $1000 \mathrm{~s} / \mathrm{mm}^{2}$ ). Slice thickness of ADC maps ranged from $3 \mathrm{~mm}$ to $8 \mathrm{~mm}$. The median time interval between FDG-PET/CT and MRI scanning in patients having undergone both examinations ( $n=30)$ was 14 days (range $0-55$ days).

\section{Image analysis}

FDG-PET/CT

The FDG-PET/CT parameters were determined by delineating the volume of interest comprising the entire tumour volume using $\mathrm{MIM}^{\bullet}$ (MIM, USA), a computer-based workstation for the visualisation, quantification, and analysis of PET/CT images. The following quantitative parameters were recorded: (1) the highest standardised uptake value (SUV) within the tumour volume of interest $\left(\mathrm{SUV}_{\max }\right)$; (2) the mean SUV within the tumour volume of interest $\left(\mathrm{SUV}_{\text {mean }}\right)$; (3) the metabolic tumour volume (MTV), which is defined as the volume of the tumour with high glycolytic activity; and (4) total lesion glycolysis (TLG), which is defined as the product of SUV mean and MTV.

MTV was calculated as the volume of hypermetabolic FDG tumour uptake, by using a gradient technique from commercially available software (PET Edge-MIM Vista software, Inc., Cleveland, Ohio). Whereas the SUVmax is the maximum derived ratio of the image derived radioactivity concentration and the whole body concentration of the injected radioactivity.

\section{MRI}

Tumour size was measured on T2-weighted MRI images in the parasagittal and para-axial planes (relative to the long axis of the cervix). Maximum tumour diameters were measured in three orthogonal planes: anteroposterior (AP), transverse (TV), and craniocaudal (CC). The largest diameter on MRI (MRI-LD) was recorded as the largest of the three tumour diameters. Tumour volume on MRI (MRI-TV) was estimated using the following equation: Tumour volume $=\mathrm{AP}$ diameter $\times \mathrm{TV}$ diameter $\times \mathrm{CC}$ diameter/2. Tumour $\mathrm{ADC}$ values were measured as the $\mathrm{ADC}_{\text {mean }}$ within a region of 
interest (ROI), which was manually drawn on the ADC map depicting the largest cross-sectional tumour diameter. The ROI (mean area $225 \mathrm{~mm}^{2}$ ) comprised a representative part of the tumour while avoiding the tumour tissue in closest proximity to adjacent normal tissue (so as to prevent normal tissues from being falsely included) and excluding necrotic or haemorrhagic areas, if present.

\section{Statistical analysis}

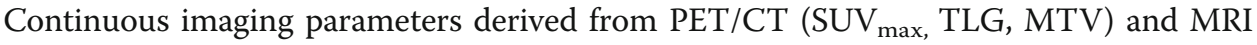
(longest lesion diameter, tumour volume and $\mathrm{ADC}_{\text {mean }}$ ) were reported as median values. Correlations between variables were calculated with Spearman correlation coefficient. Cox regression modelling was used to investigate associations between imaging markers and outcome. Survival curves were generated using the Kaplan-Meier method, and these were compared using log-rank test. Receiver-operating characteristic (ROC) curves for the prediction of progression/recurrence were used to define optimal cutoff values (based on Youden's index) for SUVmax, MTV, TLG, lesion size and tumour volume. All analyses were performed using SPSS software version 22.0 for Windows (IBM Corporation, Armonk, NY, USA).

\section{Results}

\section{Patient characteristics}

A total of 53 patients were enrolled in this study, and their median age was 50 years (Table 1). The majority of the patients had FIGO stage IIB (39\%), followed by IIIB (24\%), and $80 \%$ had squamous cell carcinoma. As per the standard of care at our institution, patients received surgery and/or chemoradiotherapy (CRT)/chemotherapy. Altogether, 18 patients (34\%) underwent radical hysterectomy; of these patients, 14 subsequently underwent postoperative CRT, whereas the remaining four patients received no adjuvant therapy. Twenty-six patients (49\%) received exclusive CRT, and nine patients (17\%) received chemotherapy alone.

\section{Patient follow-up}

At the time of the last follow-up, 35 of 53 patients (66\%) were alive without evidence of disease, 10 patients (19\%) were alive with disease, and eight patients (15\%) had died from the disease. During the study period, progression or recurrence occurred in $13 \mathrm{pa}-$ tients (24\%), and the median times to recurrence and progression were 9 months (range, 7-44 months) and 5 months (range, 2-11 months), respectively. Pelvic recurrence was observed in four patients, and extrapelvic recurrence (lung, liver, and cerebral metastases) was documented in three patients. The median time to death in patients who had died from the disease was 11 months (range, 5-32 months).

\section{Relationship between imaging parameters and clinicopathological factors}

All patients had increased FDG uptake in the cervical tumours detected at PET/CT $(n=53)$ (Figs. 1 and 2), and all patients who had also undergone MRI $(n=51)$ had a measurable cervical lesion depicted at MRI (Figs. 1 and 2). Metabolic FDG-PET/CT quantifications of the cervical lesions yielded median $\mathrm{SUV}_{\max }$, MTV, and TLG values of 17.3 (range, 4.2-51.3), $30 \mathrm{~mL}$ (range, 2-314 mL), and $246 \mathrm{~g}$ (range, 6-2417 g), respectively 

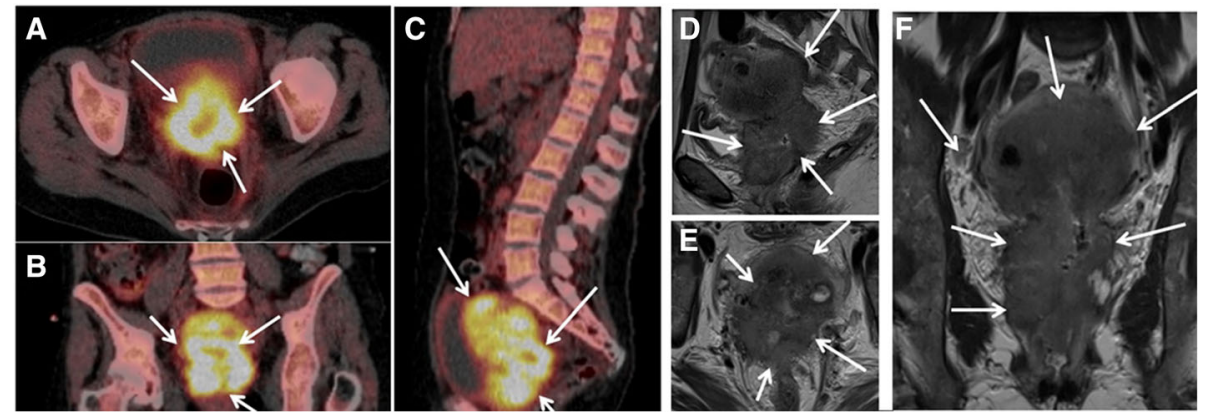

Fig. 1 A 51-year- old woman with FIGO stage IVA cervical cancer with highly FDG avid cervical tumour encircled (arrows in $\mathbf{a}, \mathbf{b}$ and $\mathbf{c}$ ) yielding a MTV of 313, TLG of 2416 and SUVmax of 16. The T2-weighted MR images show a cervical mass (arrows in $\mathbf{d}$, $\mathbf{e}$ and $\mathbf{f}$ ) extending into the bladder and with parametrial growth causing bilateral hydronephrosis. This patient died from disease two years and nine months after primary diagnosis. (a: Axial PET/CT, b: Coronal PET/CT, c: Sagittal PET/CT, d: Sagittal T2-weighted MRI, e: Axial T2-weighted MRI and $\mathbf{f}$ : Coronal T2-weighted MRI)

(Table 2). The MRI-LD was $44 \mathrm{~mm}$ (range, 9-112 mm), and the MRI-TV was $25 \mathrm{~mL}$ (range, 0.3-310 mL; Table 2). All metabolic tumour parameters from PET/CT (SUV $\max$, TLG, and MTV) were positively correlated with the tumour size parameters from MRI (MRI-LD and MRI-TV), yielding $\mathrm{r}_{\mathrm{s}} \geq 0.29$ and $P \leq 0.04$ for all correlations (Table 3). Among the metabolic tumour parameters, TLG was the most positively correlated with the MRI-based tumour size parameters $\left(\mathrm{r}_{\mathrm{s}} \geq 0.77\right.$ and $P<0.001$ for both; Table 3$)$.

Furthermore, high values of MTV, TLG, MRI-LD, and MRI-TV were associated with high clinical FIGO stage $(P \leq 0.005$ for all), whereas no significant association was observed between the $\mathrm{SUV}_{\text {max }}$ and FIGO stage $(P=0.315$; Table 3$)$.

\section{Relationship between FDG-PET/CT imaging parameters and clinicopathological factors}

All patients had increased FDG uptake in the cervical tumours detected at PET/CT $(n=53)$ (Figs. 1 and 2). Metabolic FDG-PET/CT quantifications of the cervical lesions yielded
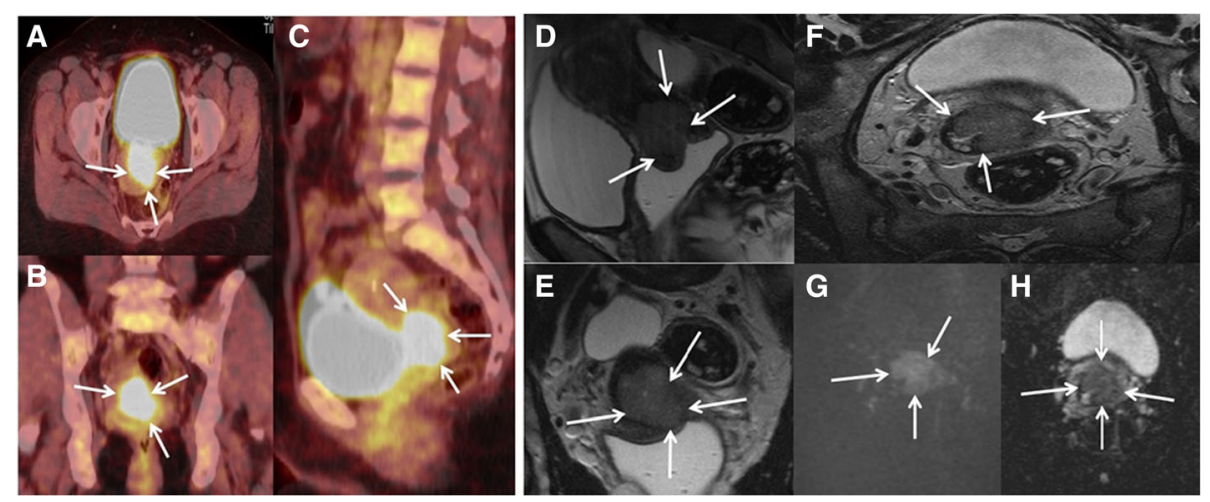

Fig. 2 A 47-year- old woman with FIGO stage IIB cervical cancer with FDG avid cervical tumour encircled (arrows in $\mathbf{a}, \mathbf{b}$ and $\mathbf{c}$ ) yielding a MTV of 20, TLG of 170 and SUVmax of 16. The T2-weighted MR images show a cervical tumour (arrows in $\mathbf{d}$, e and $\mathbf{f}$ ) and corresponding diffusion-restriction in b800 DWI (g) and ADC map (h). This patient was alive with no signs of cervical cancer at follow-up date nine months after primary treatment. (a: Axial PET/CT, b: Coronal PET/CT, c: Sagittal PET/CT, d: Sagittal T2-weighted MRI, e: Coronal T2-weighted MRI and f: Axial T2-weighted MRI) 
Table 2 Median (range) tumour values of FDG-PET/CT and MRI parameters

\begin{tabular}{lll}
\hline Variable & Median & Range \\
\hline SUVmax & 17.3 & $4.2-51.3$ \\
MTV, $\mathrm{mL}$ & 30 & $2.0-314$ \\
TLG, $\mathrm{g}$ & 246 & $6.0-2417$ \\
MRI LD, $\mathrm{mm}$ & 44 & $9.0-112$ \\
MRI TV, $\mathrm{mL}$ & 25 & $0.3-310$ \\
$\mathrm{ADC}, 10^{-6} \mathrm{~mm}^{2} / \mathrm{s}$ & 715 & $491-1020$
\end{tabular}

$A D C$ Apparent diffusion coefficient, $M R I-L D$ Longest diameter of the tumour measured by magnetic resonance imaging, MRI-TV Tumour volume measured by magnetic resonance imaging, MTV Metabolic tumour volume, SUVmax Maximum standardised uptake value and TLG Total lesion glycolysis

median $\mathrm{SUV}_{\text {max }}$, MTV, and TLG values of 17.3 (range, 4.2-51.3), $30 \mathrm{~mL}$ (range, 2-314 mL), and $246 \mathrm{~g}$ (range, 6-2417 g), respectively (Table 2). Furthermore, high values of MTV, TLG, were associated with high clinical FIGO stage $(P \leq 0.005)$, whereas no significant association was observed between the $\mathrm{SUV}_{\max }$ and FIGO stage $(P=0.315$; Table 3$)$.

\section{Relationship between MRI imaging parameters and clinicopathological factors}

All patients who had also undergone MRI $(n=51)$ had a measurable cervical lesion depicted at MRI (Figs. 1 and 2). The MRI-LD was $44 \mathrm{~mm}$ (range, 9-112 $\mathrm{mm}$ ), and the MRI-TV was $25 \mathrm{~mL}$ (range, $0.3-310 \mathrm{~mL}$; Table 2). Additionally, high values of MRI-LD, and MRI-TV were associated with high clinical FIGO stage $(P \leq 0.005)$.

\section{Relationship between FDG-PET/CT and MRI imaging parameters}

All metabolic tumour parameters from PET/CT (SUV $\max$, TLG, and MTV) were positively correlated with the tumour size parameters from MRI (MRI-LD and MRI-TV), yielding $\mathrm{r}_{\mathrm{s}} \geq 0.29$ and $P \leq 0.04$ for all correlations (Table 3). Among the metabolic tumour parameters, TLG was the most positively correlated with the MRI-based tumour size parameters $\left(\mathrm{r}_{\mathrm{s}} \geq 0.77\right.$ and $P<0.001$ for both; Table 3$)$.

Table 3 Correlation between PET/CT parameters, MRI parameters and FIGO stage

\begin{tabular}{|c|c|c|c|c|c|c|c|c|c|c|c|c|c|}
\hline \multirow[t]{2}{*}{ Variable } & SUVmax & \multicolumn{2}{|l|}{ TLG } & \multicolumn{2}{|l|}{ MTV } & \multicolumn{2}{|c|}{ MRI-LD } & \multicolumn{2}{|c|}{ MRI-TV } & \multicolumn{2}{|l|}{$A D C$} & \multicolumn{2}{|l|}{ FIGO } \\
\hline & $\begin{array}{ll}r_{s} & p^{a}\end{array}$ & $\overline{r_{s}}$ & $p^{a}$ & $r_{s}$ & $p^{a}$ & $r_{s}$ & $p^{a}$ & $r_{s}$ & $p^{a}$ & $\overline{r_{5}}$ & $p^{a}$ & $r_{s}$ & $p^{*}$ \\
\hline SUVmax & & 0.66 & $<0.001$ & 0.46 & $<0.001$ & 0.29 & 0.040 & 0.36 & 0.010 & -0.18 & 0.34 & 0.14 & 0.32 \\
\hline TLG & & & & 0.89 & $<0.001$ & 0.77 & $<0.001$ & 0.82 & $<0.001$ & 0.10 & 0.60 & 0.43 & 0.001 \\
\hline MTV & & & & & & 0.72 & $<0.001$ & 0.74 & $<0.001$ & 0.21 & 0.26 & 0.38 & 0.005 \\
\hline MRI-LD & & & & & & & & 0.96 & $<0.001$ & 0.16 & 0.38 & 0.48 & $<0.001$ \\
\hline MRI-TV & & & & & & & & & & 0.18 & 0.33 & 0.45 & $<0.001$ \\
\hline$A D C$ & & & & & & & & & & & & 0.22 & 0.24 \\
\hline FIGO & & & & & & & & & & & & & \\
\hline
\end{tabular}

${ }^{a}$ Spearman's bivariate correlation test Significant $p$ values $(<0.05)$ are given in bold

$A D C$ Apparent diffusion coefficient, FIGO International Federation of Gynaecology and Obstetrics, MRI-LD Longest diameter of the tumour measured by magnetic resonance imaging, MRI-TV Tumour volume measured by magnetic resonance imaging, MTV Metabolic tumour volume, SUVmax Maximum standardised uptake value and TLG Total lesion glycolysis 
Relation between $A D C_{\text {mean }}$ and quantitative FDG-PET/CT and MRI parameters or FIGO stage The median tumour $\mathrm{ADC}_{\text {mean }}$ value was $715 \times 10^{-6} \mathrm{~mm}^{2} / \mathrm{s}$ (range, 491-1020 $\times$ $\left.10^{-6} \mathrm{~mm}^{2} / \mathrm{s}\right)$ in the subgroup of patients who had undergone DW-MRI $(n=30$; Table 2). No significant correlations were observed between the tumour $\mathrm{ADC}_{\text {mean }}$ and the other imaging parameters or FIGO stage (Table 3).

\section{Cutoff value of imaging parameters for predicting progression/recurrence}

Among the derived imaging parameters, SUVmax yielded the lowest area under curve (AUC) with AUC $=0.67(P=0.06)$, whereas MTV and TLG yielded the highest AUC with AUC $=0.80(P<0.001)$ and AUC $=0.76(P<0.006)$, respectively, for predicting recurrence or progression (Fig. 3). For MRI-LD and MRI-TV the corresponding figures were $\mathrm{AUC}=0.69, P<0.044$ and AUC $=0.69, P<0.047$, respectively.

The optimal cervical lesion cutoff values for SUVmax, MTV, TLG, MRI-LD, and MRI-TV for predicting recurrence/progression were 16.0, $56.7 \mathrm{~mL}, 412 \mathrm{~g}, 37.5 \mathrm{~mm}$, and $36.2 \mathrm{~mL}$, respectively.

\section{Survival analysis}

MTV $\geq 56.7 \mathrm{~mL}, T L G \geq 412 \mathrm{~g}$, advanced FIGO stage ( $\geq$ IIIA) and MRI-TV $\geq 36.0 \mathrm{~mL}$ were significantly associated with progression/recurrence-free survival in univariable analyses $(P=0.004, P=0.002, P=0.004$, and $P=0.022$, respectively; Table 4$)$. The corresponding Kaplan-Meier survival curves based on MTV ( $\geq 56.7 \mathrm{~mL}$ vs $<56.7 \mathrm{~mL}$ ) and

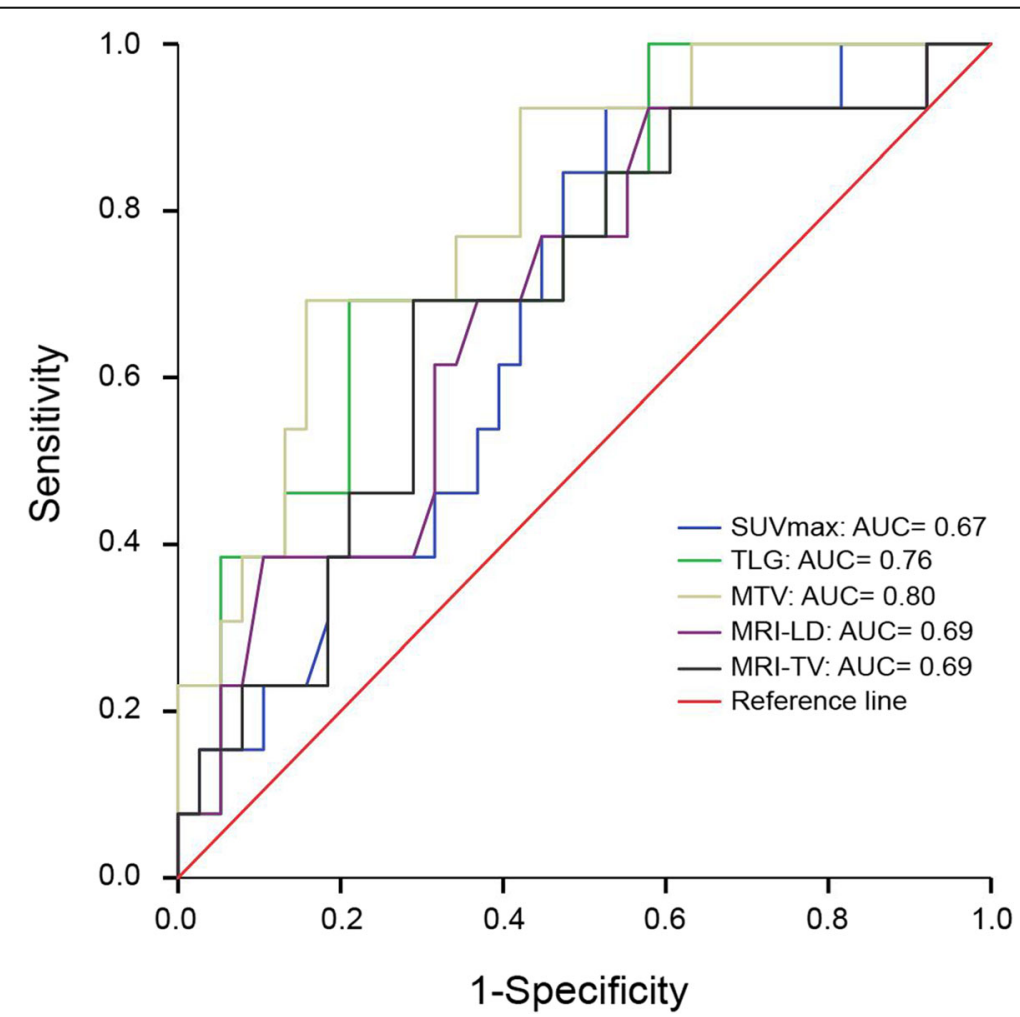

Fig. 3 Receiver operating characteristic curve analyses of MTV, TLG, MRI-LD and MRI-TV values for predicting progression and recurrence 
Table 4 Univariable and multivariable cox-regression analyses for PET-CT and MRI parameters and FIGO stage for predicting progression/recurrence free survival in cervical cancer patients

\begin{tabular}{|c|c|c|c|c|c|c|c|}
\hline \multirow[t]{2}{*}{ Variables } & \multirow{2}{*}{$\begin{array}{l}\text { Test of Progression/ } \\
\text { Recurrence free survival }\end{array}$} & $\mathrm{HR}$ & $95 \% \mathrm{Cl}$ & \multirow[t]{2}{*}{$P$-value } & $H R$ & $95 \% \mathrm{Cl}$ & \multirow[t]{2}{*}{$P$-value } \\
\hline & & \multicolumn{2}{|c|}{ Univariable } & & \multicolumn{2}{|c|}{ Multivariable } & \\
\hline SUVmax & $\geq 16.0$ vs. $<16.0$ & 4.2 & $0.5-32.9$ & 0.17 & & & \\
\hline MTV & $\geq 56.7 \mathrm{~mL}$ vs. $<56.7 \mathrm{~mL}$ & 7.8 & $2.1-29.5$ & 0.002 & 7.8 & $1.2-47.6$ & 0.025 \\
\hline TLG & $\geq 412 \mathrm{~g}$ vs. $<412 \mathrm{~g}$ & 6.9 & $1.8-26.0$ & 0.004 & 7.5 & $0.7-80.7$ & 0.09 \\
\hline FIGO stage & $\geq \|$ A vs. $<\| B$ & 6.9 & $1.8-26.47$ & 0.004 & 5.5 & $1.3-23.0$ & 0.020 \\
\hline MRI LD & $\geq 37.6$ vs. $<37.6 .0$ & 5.2 & $0.6-40.4$ & 0.11 & & & \\
\hline MRI TV & $\geq 36.0 \mathrm{~mL}$ vs. $<36.0 \mathrm{~mL}$ & 4.6 & $1.2-17.0$ & 0.022 & 0.6 & $0.1-4.1$ & 0.66 \\
\hline
\end{tabular}

Significant $p$-values are given in bold for univariable and multivariable analysis

FIGO International Federation of Gynaecology and Obstetrics, MRI-LD Longest diameter of the tumour measured by magnetic resonance imaging, MRI-TV Tumour volume measured by magnetic resonance imaging, MTV Metabolic tumour volume, SUVmax Maximum standardised uptake value and TLG Total lesion glycolysis

FIGO stage ( $\geq$ IIIA vs $\leq$ IIB) are given in Fig. 4. Lesion SUVmax $\geq 16.0$ and MRI-LD $\geq 37.6 \mathrm{~mm}$ did not significantly predict progression/recurrence-free survival $(P=0.17$ and 0.11 , respectively; Table 4$)$.

In the multivariable model of the significant parameters, MTV and TLG were included in two separate models, as these two parameters are inherently strongly correlated with each other (since TLG is derived from MTV). High MTV $(\geq 56.7 \mathrm{~mL}$; hazard ratio $(\mathrm{HR})=7.8 ; P=0.025)$ and advanced FIGO stage ( $\geq$ IIIA; $\mathrm{HR}=5.5 ; P=0.020$ ) were the only factors independently predicting poor prognosis in the multivariable analyses, whereas MRI-TV $(\geq 36.0 \mathrm{~mL} ; \mathrm{HR}=0.6 ; P=0.65)$ and TLG ( $\geq 412 \mathrm{~g} ; \mathrm{HR}=7.5 ; P=0.093)$ did not reach statistical significance (Table 4 ).

\section{Discussion}

In this study, we have demonstrated the prognostic value of metabolic tumor parameters based on preoperative FDG-PET/CT in cervical carcinoma and found that these imaging parameters are reflected in clinical staging parameters. We found that high MTV independently predicts poor prognosis in cervical cancer patients. MTV is thus a very promising imaging biomarker, potentially aiding in prognostication and treatment planning in uterine cervical cancer.
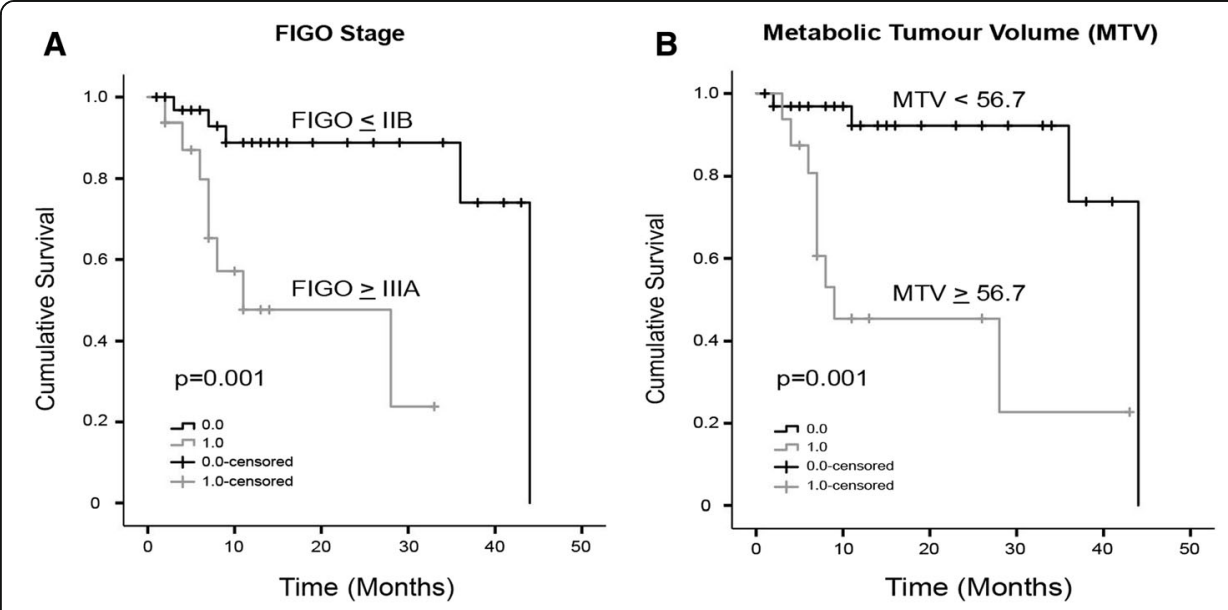

Fig. 4 Kaplan-Meier survival curves of progression/recurrence according to FIGO stage (a) and MTV 56.7 (b) 
In this study, we found the optimal cutoff value of MTV for prognostication to be $56.7 \mathrm{~mL}$. This is somewhat in line with recently published literature. Chung et al. (2011) reported that preoperative MTV $\geq 23.4 \mathrm{~mL}$ independently predicted poor survival in cervical cancer patients stage IB-IIA who were treated with radical surgery, and that high MTV was associated with advanced FIGO stage, parametrial involvement, and lymph node metastases. Recently, Hong et al. (2016) evaluated the prognostic value of MTV in patients with cervical cancer treated with definitive chemoradiotherapy. In their study, MTV $>47.8 \mathrm{~mL}$ was found to significantly predict reduced recurrence free survival.

Lai et al. (2017) compared FDG PET/CT metabolic features and MRI features in cervical cancer. This very recent study reported a very strong association between MTV and anatomical tumour volume (based on T2W MRI) and suggested MTV as a promising imaging biomarker, superior to DW-MRI-based features. In our study, MTV was not only correlated with lesion size but also with FIGO stage, whereas $\mathrm{SUV}_{\text {max }}$ in the primary tumour was not significantly associated with FIGO stage. MTV represents the entire tumor volume being metabolically active whereas $\mathrm{SUV}_{\text {max }}$ represents the single tumor voxel exhibiting the highest SUV. This may explain our finding that MTV seems superior to $\mathrm{SUV}_{\max }$ in reflecting the true tumour burden and also for predicting FIGO stage in cervical cancer.

Interestingly, high $\mathrm{SUV}_{\max }$ of the primary tumour also failed to predict reduced progression/recurrence free survival $(P=0.133)$ in cervical cancer patients. This finding is in line with previously published studies. Yoo et al. (2012) reported that the $\mathrm{SUV}_{\max }$ of the primary tumour was not correlated with recurrence-free survival. In addition, they suggested MTV as a better predictor of prognosis than other FDG-PET parameters. Similar results were reported by Akkas et al. (2013), who stated that the tumour SUVmax was not an independent prognostic factor for predicting disease recurrence in patients with inoperable cervical cancer. In another recent study, Crivellaro et al. (2012) prospectively analysed 69 cervical cancer patients with FIGO stage IB1-IIA and found that SUVmax of the primary tumor did not predict recurrence in early stage cervical cancer. Moreover, a recent study demonstrated that $\mathrm{SUV}_{\max }$ of the primary tumor was not found to be predictive of overall survival $(P=0.233)$ (Chong et al., 2015). Hence, findings from these reports and the present study illustrate that although tumour SUVmax is widely used as a prognostic marker for several malignancies, it has obvious limitations that may be due to several factors. First, $\mathrm{SUV}_{\max }$ refers to the single highest metabolic voxel, which may not be representative of the metabolic activity of the entire tumour. Second, larger tumours are not necessarily more metabolically active than smaller tumours. Third, the calculated tumour $\mathrm{SUV}_{\max }$ is inherently dependent on several technical aspects (image reconstruction method, iterative algorithm, and PET scanner) and biological factors (serum glucose levels and body weight) that may reduce the transferability of proposed cutoff values between patients and scanners.

MRI is the mainstay for staging of pelvic tumours and DW-MRI is increasingly employed to characterise the tumour microenvironment and tumour microstructure. Tumour ADC values derived from DW-MRI have been shown to yield functional information about tumour tissue and to be negatively correlated with tumour cellularity. Furthermore, since tumour ADC values are typically lower than those of adjacent healthy tissues, DW-MRI may aid in tumour delineation, tumour characterisation, and evaluation of treatment response (Le Bihan, 2013). 
In the present study, no association was observed between tumour ADC and $\mathrm{SUV}_{\max }$ values or between the ADC and MTV or TLG values. Regarding the overall correlations between these parameters, the literature describes inconsistent results for the association between the ADC and absolute $\mathrm{SUV}_{\max }$ values derived from stand-alone MRI and separate hybrid PET/CT scanners. Brandmaier et al. (2015) evaluated the possible correlation between the ADC and $\mathrm{SUV}_{\max }$ values in patients with primary $(n=14)$ and recurrent tumours $(n=17)$ of the cervix uteri. They identified a significant inverse correlation between the $\mathrm{ADC}_{\text {mean }}$ and $\mathrm{SUV}_{\text {max }}$ values $(r=-0.53, P=0.05)$ in primary tumours and recurrent local tumours $(r=-0.74, P=0.002)$. Similarly, Grueneisen et al. (2014) investigated the potential correlation between the $\mathrm{SUV}_{\max }$ and $\mathrm{ADC}_{\text {mean }}$ values in primary and recurrent cervical cancer and confirmed a significant inverse correlation $(r=-0.62, P<0.05)$. However, Ho et al. (2009) reported no association between the pretreatment $\mathrm{SUV}_{\max }$ and $\mathrm{ADC}_{\text {mean }}$ values in primary cervical cancer $(n=33)$. Furthermore, care must be taken while reporting $\mathrm{ADC}$ values because ADC quantification is dependent on the chosen b-values, scanner geometry, tissue perfusion, and field strength (Kallehauge et al., 2010).

In the era of radiomics, the spectra and complexity of image based quantitative measurements are rapidly increasing. Assessments of tumour heterogeneity, in different imaging modalities, have gained a lot of attention. In cervical cancer, Lucia et al. (2018) recently presented a pretreatment risk stratification model based on FDG-PET/CTand MRI-based measurements, in which grey level non-uniformity in PET images and entropy in ADC maps were independent prognostic factors. Although further validation is needed, such findings pinpoint that traditional tumour measurements might be outperformed - and eventually replaced by radiomics approaches.

Given the complexity of the female pelvic anatomy, hybrid PET/MRI allows simultaneous imaging exclusively in which the two modalities are truly synergistic, which allows better understanding of tumor biology and morphology. In a recent study by Queiroz et al. (2015) on various gynecological cancer patients $(n=26)$, the authors concluded that the T-staging accuracy by FDG-PET/MRI was superior to that of PET-CT $(P<0.001)$. Also Kitajima et al. (2014) found that fused PET/MRI outperformed PET/CT with corresponding accuracies of 83.3 and 53.3, respectively, for T-staging in cervical cancer $(n=30)$. Similarly, Nakajo et al.(2010) reported that fused PET/MRI was superior to PET/CT for the cervical cancer detection and delineation. PET/MRI does also have a very promising role for assessing therapeutic response and for detecting disease recurrence (Sarabhai et al., 2018). Interestingly, hybrid PET/MRI imaging also increases the radiologists' diagnostic confidence in detecting gynecological malignant lesions when compared to FDG-PET/CT or stand-alone MRI (Grueneisen et al., 2014; Beiderwellen et al., 2014).

Thus, the potential advantages of PET/MRI in a clinical setting are 1) better understanding of tumor biology and morphology; 2) better characterization of tumour and its adjacent structures aiding to distinguish nodal involvement from physiologic metabolic activity within normal structures; 3 ) improved local staging accuracy due to superior soft tissue contrast; 4) better detection of metastatic disease by DWI in organs having high FDG-PET background uptake (e.g. brain and liver); and 5) reduction in radiation dose exposure (by omitting $\mathrm{CT}$ ) in a vulnerable patient population. To this end, hybrid PET/MRI imaging seems to be highly feasible and favourable in gynaecological cancer patients. However, the full potential of this relatively new imaging modality needs to be further investigated in future large prospective clinical trials. 
This study has some limitations. First, this is a retrospective study with a limited number of patients, particularly those with DW-MRI measurement. Second, the follow-up duration was relatively short. Third, our study population was heterogeneous, with patients having different clinical FIGO stages and undergoing different treatment modalities. However, we evaluated the potential of pretreatment prognostic biomarkers, which are by definition independent of any treatment received. Finally, the fact that the MRI protocols and particularly the DW-MRI were not standardized in our study may have limited the evaluation of DW-MRI; however reflecting a routine setting not interfering with the diagnostic work-up in cervical cancer.

Furthermore, we used a commercially available automated tumour-delineating software algorithm. Therefore, the MTV measurement method in this study is likely to be reliable and reproducible, although not tested for inter- or intraobserver reproducibility, and may be easily employed in the clinical setting. Validation of the results of the present study in independent patient populations is, however, necessary before generalisations can be made. Prospective clinical studies with a larger number of patients and with longer follow-up periods will be needed to validate the present findings.

\section{Conclusion}

In conclusion, this study demonstrated that in patients with cervical cancer, pretreatment large primary tumour MTV derived from FDG-PET/CT is an independent prognostic biomarker predicting reduced progression/recurrence-free survival and an aggressive tumour phenotype. PET/CT based MTV measurements may help clinicians identify high-risk cervical cancer patients that may be candidates for more frequent follow-ups or more aggressive treatment strategies in future clinical trials.

Acknowledgements

"The publication of this article was supported by funds of the European Association of Nuclear Medicine (EANM)".

Authors' contributions

Study conception and design: V.R.B., S.Y., and I.S.H. Acquisition of data: S.Y., A.G., and M.K.H. Analysis and interpretation of data: V.R.B., S.Y., A.G., Ø.S., M.K.H and I.S.H. Drafting of manuscript: V.R.B., S.Y., C.K., J.T and I.S.H. Critical revision: K.W.,

J.T., C.K., Ø.S and I.S.H. All authors read and approved the final manuscript.

Ethics approval and consent to participate

Retrospective study - For this type of study formal consent is not required.

Informed consent: Informed consent was obtained from all individual participants included in the study.

Consent for publication

Not applicable.

Competing interests

The authors declare that they have no competing interests.

\section{Publisher's Note}

Springer Nature remains neutral with regard to jurisdictional claims in published maps and institutional affiliations.

\section{Author details}

'Department of Obstetrics and Gynecology, Haukeland University Hospital, Bergen, Norway. ${ }^{2}$ Centre for Cancer Biomarkers, Department of Clinical Science, University of Bergen, Bergen, Norway. ${ }^{3}$ Department of Radiology, Ziekenhuis Oost-Limburg, Campus St-Jan, Genk, Belgium. ${ }^{4}$ Mohn Medical Imaging and Visualization Centre (MMIV), Department of Radiology, Haukeland University Hospital, Bergen, Norway. ${ }^{5}$ Department of Clinical Medicine, University of Bergen, Bergen, Norway. ${ }^{6}$ Unit for Applied Clinical Research, Department of Cancer Research and Molecular Medicine, Norwegian University of Science and Technology, Trondheim, Norway. 
Received: 21 March 2018 Accepted: 3 September 2018

\section{Published online: 11 December 2018}

\section{References}

Akkas BE, Demirel BB, Dizman A, Vural GU (2013) Do clinical characteristics and metabolic markers detected on positron emission tomography/computerized tomography associate with persistent disease in patients with in-operable cervical cancer? Ann Nucl Med 27(8):756-763. https://doi.org/10.1007/s12149-013-0745-1

Balleyguier C, Sala E, Da Cunha T et al (2011) Staging of uterine cervical cancer with MRI: guidelines of the European Society of Urogenital Radiology. Eur Radiol 21(5):1102-1110. https://doi.org/10.1007/s00330-010-1998-x

Beiderwellen K, Huebner M, Heusch P et al (2014) Whole-body [18F]FDG PET/MRI vs. PET/CT in the assessment of bone lesions in oncological patients: initial results. Eur Radiol 24(8):2023-2030. https://doi.org/10.1007/s00330-014-3229-3

Benedet JL, Bender H, Jones H, Ngan HY, Pecorelli S (2000) FIGO staging classifications and clinical practice guidelines in the management of gynecologic cancers. FIGO committee on gynecologic oncology. Int J Gynaecol Obstet 70(2):209-262. https://www.ncbi.nlm.nih.gov/pubmed/11041682. Accessed 19 Feb 2017

Boellaard R, Delgado-Bolton R, Oyen WJG et al (2015) FDG PET/CT: EANM procedure guidelines for tumour imaging: version 2.0. Eur J Nucl Med Mol Imaging 42(2):328-354. https://doi.org/10.1007/s00259-014-2961-x

Bollineni VR, Kramer G, Liu Y, Melidis C, deSouza NM (2015) A literature review of the association between diffusion-weighted MRI derived apparent diffusion coefficient and tumour aggressiveness in pelvic cancer. Cancer Treat Rev 41(6):496-502. https://doi.org/10.1016/j.ctrv.2015.03.010

Brandmaier P, Purz S, Bremicker K, et al. Simultaneous [18F]FDG-PET/MRl: correlation of apparent diffusion coefficient (ADC) and standardized uptake value (SUV) in primary and recurrent cervical Cancer. Bathen TF, ed. PLoS One 2015;10(11): e0141684. doi:https://doi.org/10.1371/journal.pone.0141684

Choi HJ, Kim SH, Seo S-S et al (2006) MRI for pretreatment lymph node staging in uterine cervical Cancer. Am J Roentgenol 187(5):W538-W543. https://doi.org/10.2214/AJR.05.0263

Chong GO, Jeong SY, Park S-H et al (2015) Comparison of the prognostic value of F-18 pet metabolic parameters of primary tumors and regional lymph nodes in patients with locally advanced cervical Cancer who are treated with concurrent Chemoradiotherapy. PLoS One 10(9):e0137743. https://doi.org/10.1371/journal.pone.0137743 Li JJ, ed

Chung HH, Kim JW, Han KH et al (2011) Prognostic value of metabolic tumor volume measured by FDG-PET/CT in patients with cervical cancer. Gynecol Oncol 120(2):270-274. https://doi.org/10.1016/j.ygyno.2010.11.002

Cibula D, Pötter R, Raspollini MR. ESGO-ESTRO-ESP guidelines on the management of invasive cervical cancer. 2017. https://guidelines.esgo.org/media/2018/04/ESGO_Cervical-Cancer_A6.pdf

Crivellaro C, Signorelli M, Guerra L et al (2012) 18F-FDG PET/CT can predict nodal metastases but not recurrence in early stage uterine cervical cancer. Gynecol Oncol. https://doi.org/10.1016/j.ygyno.2012.06.041

Grueneisen J, Beiderwellen K, Heusch P et al (2014) Correlation of standardized uptake value and apparent diffusion coefficient in integrated whole-body PET/MRI of primary and recurrent cervical Cancer. PLoS One 9(5):e96751. https://doi.org/10.1371/journal.pone.0096751 Hoffmann A-C, ed

Ho K-C, Lin G, Wang J-J, Lai C-H, Chang C-J, Yen T-C (2009) Correlation of apparent diffusion coefficients measured by 3 T diffusion-weighted MRI and SUV from FDG PET/CT in primary cervical cancer. Eur J Nucl Med Mol Imaging 36(2):200-208. https://doi.org/10.1007/s00259-008-0936-5

Hong JH, Min KJ, Lee JK et al (2016) Prognostic value of the sum of metabolic tumor volume of primary tumor and lymph nodes using 18F-FDG PET/CT in patients with cervical Cancer. Medicine (Baltimore). https://doi.org/10.1097/MD. 0000000000002992

Howlader N, Noone AM, Krapcho M, et al (2011) SEER Cancer Statistics Review, 1975-2008. National Cancer Institut, Bethesda. https://seer.cancer.gov/csr/1975_2008/. Accessed 26 Mar 2013

Kallehauge JF, Tanderup K, Haack S et al (2010) Apparent diffusion coefficient (ADC) as a quantitative parameter in diffusion weighted MR imaging in gynecologic cancer: dependence on b-values used. Acta Oncol (Madr) 49(7):1017-1022. https://doi.org/10.3109/0284186X.2010.500305

Kitajima K, Suenaga Y, Ueno Y et al (2014) Fusion of PET and MRI for staging of uterine cervical cancer: comparison with contrastenhanced 18 F-FDG PET/CT and pelvic MRI. Clin Imaging 38(4):464-469. https://doi.org/10.1016/j.clinimag.2014.02.006

Lai AYT, Perucho JAU, Xu X, Hui ES, Lee EYP (2017) Concordance of FDG PET/CT metabolic tumour volume versus DW-MRI functional tumour volume with T2-weighted anatomical tumour volume in cervical cancer. BMC Cancer 17(1):825. https://doi.org/10.1186/s12885-017-3800-9

Le Bihan D (2013) Apparent diffusion coefficient and beyond: what diffusion MR imaging can tell us about tissue structure. Radiology 268(2):318-322. https://doi.org/10.1148/radiol.13130420

Lucia F, Visvikis D, Desseroit M-C et al (2018) Prediction of outcome using pretreatment 18F-FDG PET/CT and MRI radiomics in locally advanced cervical cancer treated with chemoradiotherapy. Eur J Nucl Med Mol Imaging 45(5):768-786. https://doi.org/10.1007/s00259-017-3898-7

Lv K, Guo H, Lu Y, Wu Z, Zhang K, Han J (2014) Role of 18F-FDG PET/CT in detecting pelvic lymph-node metastases in patients with early-stage uterine cervical cancer. Nucl Med Commun 35(12):1204-1211. https://doi.org/10.1097/MNM. 0000000000000198

McVeigh PZ, Syed AM, Milosevic M, Fyles A, Haider MA (2008) Diffusion-weighted MRI in cervical cancer. Eur Radiol 18(5): 1058-1064. https://doi.org/10.1007/s00330-007-0843-3

Miccò M, Vargas HA, Burger IA et al (2014) Combined pre-treatment MRI and 18F-FDG PET/CT parameters as prognostic biomarkers in patients with cervical cancer. Eur J Radiol 83(7):1169-1176. https://doi.org/10.1016/j.ejrad.2014.03.024

Mirpour S, Mhlanga JC, Logeswaran P, Russo G, Mercier G, Subramaniam RM (2013) The role of PET/CT in the Management of Cervical Cancer. Am J Roentgenol 201(2):W192-W205. https://doi.org/10.2214/AJR.12.9830

Nakajo K, Tatsumi M, Inoue A et al (2010) Diagnostic performance of fluorodeoxyglucose positron emission tomography/ magnetic resonance imaging fusion images of gynecological malignant tumors: comparison with positron emission tomography/computed tomography. Jpn J Radiol 28(2):95-100. https://doi.org/10.1007/s11604-009-0387-3

Nakamura K, Joja I, Nagasaka T et al (2012) The mean apparent diffusion coefficient value (ADCmean) on primary cervical cancer is a predictive marker for disease recurrence. Gynecol Oncol 127(3):478-483. https://doi.org/10.1016/j.ygyno.2012.07.123 
Queiroz MA, Kubik-Huch RA, Hauser N et al (2015) PET/MRI and PET/CT in advanced gynaecological tumours: initial experience and comparison. Eur Radiol 25(8):2222-2230. https://doi.org/10.1007/s00330-015-3657-8

RSM L, Ramdave S, Beech P et al (2016) Utility of SUVmax on 18 F-FDG PET in detecting cervical nodal metastases. Cancer Imaging 16(1):39. https://doi.org/10.1186/s40644-016-0095-z

Sarabhai T, Schaarschmidt BM, Wetter A et al (2018) Comparison of 18F-FDG PET/MRI and MRI for pre-therapeutic tumor staging of patients with primary cancer of the uterine cervix. Eur J Nucl Med Mol Imaging 45(1):67-76. https://doi.org/10. 1007/s00259-017-3809-y

Sironi S, Buda A, Picchio M et al (2006) Lymph node metastasis in patients with clinical early-stage cervical Cancer: detection with integrated FDG PET/CT. Radiology 238(1):272-279. https://doi.org/10.1148/radiol.2381041799

Yoo J, Choi JY, Moon SH et al (2012) Prognostic significance of volume-based metabolic parameters in uterine cervical Cancer determined using 18F-Fluorodeoxyglucose positron emission tomography. Int J Gynecol Cancer 22(7):1226-1233. https://doi.org/10.1097/IGC.0b013e318260a905

Submit your manuscript to a SpringerOpen ${ }^{\odot}$ journal and benefit from:

- Convenient online submission

- Rigorous peer review

- Open access: articles freely available online

- High visibility within the field

- Retaining the copyright to your article

Submit your next manuscript at $\boldsymbol{\nabla}$ springeropen.com 